\title{
Effects of two different somatostatin analogs on glucose tolerance in acromegaly
}

\author{
C. Ronchi, P. Epaminonda, V. Cappiello, P. Beck-Peccoz, and M. Arosio \\ Institute of End ocrine Sciences, University of M ilan, O spedale Maggiore IRCCS, M ilan, Italy
}

\begin{abstract}
Impaired glucose tolerance is present in many acromegalic patients and treatment with somatostatin analogs has variable effects on glycemic control. The aim of this study was to compare the effects of 2 somatostatin analogs on glucose metabolism, lanreotide slow release (L-SR) and octreotide long acting release (O-LAR), in 10 patients with acromegaly ( 2 of whom with overt Type 2 diabetes mellitus). Glucose and insulin levels in fasting conditions and in response to OGTT, evaluated as AUC, insulin resistance (IR) evaluated by homeostatic model assessment (HOMA-IR), glycosylated hemoglobin $\left(\mathrm{HbA}_{1 c}\right)$, GH, IGF-I, were assessed during L-SR and O-LAR treatment. Mean fasting glucose, glucose response to OGTT and $\mathrm{HbA}_{1 \mathrm{c}}$ levels in 8 non-diabetic patients did not significantly change after L-SR therapy while they all increased after O-LAR
\end{abstract}

\section{INTRO DUCTION}

The relationship between $\mathrm{GH}$ hypersecretion and carbohydrate metabolism is complex and not completely understood. There is a high prevalence of impaired glucose tolerance (35-50\%) and overt diabetes mellitus $(9-23 \%)$ in patients with active acromegaly, mainly due to insulin resistance (IR) (1-3).

Somatostatin analogs therapy is today the medical treatment of choice for acromegaly. It has divergent effects on glucose metabolism, depending on inhibition of $\mathrm{GH}$ and glucag on secretion, which may improve insulin action, and on suppression of insulin release itself. Results of previous studies on octreotide administration with daily multiple injections showed an improvement in whole body insulin sensitivity with variable effects on glucose lev-

Key-words: O ctreotide, lanreotide, glucose metabolism, insulin resistance, IGF-I.

Correspondence: Dr. Maura Arosio, Istituto di Scienze Endocrine, Pad. Granelli, O spedale Maggiore, IRCCS, Via F. Sforza 35, 20122 Milano, Italy. E-mail: maura.arosio@unimi.it

Accepted December 18, 2001. treatment ( $p<0.05$ vs baseline and L-SR). Mean HOMA-IR values calculated in acromegalic patients before medical therapy were higher than in normal subjects $(p<0.005)$ and showed a significant decrease during both treatments $(p<0.05)$. In the 2 diabetic acromegalic patients a worsening in glucose metabolism was observed during O-LAR treatment but not during L-SR. GH and IGF-I levels significantly decreased with both drugs and normalized respectively in 38\% and $12 \%$ with L-SR, $50 \%$ and $25 \%$ with O-LAR. In conclusion, both drugs decreased IR in acromegalic patients; O-LAR seems to be more detrimental to glucose metabolism than L-SR, despite being more effective in reducing GH and IGF-I levels. (J. Endocrinol. Invest. 25: 502-507, 2002)

○2002, Editrice Kurtis

els. Different effects have been reported in diabetic acromegalic patients (4-7).

Recently 2 new long acting release formulations of somatostatin analogs have been developed, lanreotide-slow release (L-SR) and octreotide-long acting release (O-LAR). Both drugs have been shown to be effective in lowering GH and IGF-I (8-13) and offer a considerable improvement in compliance of acromegalic patients, as the drugs need to be administered im every 7-14 days (L-SR) or every 28 days ( $O-L A R)$, respectively. Studies comparing the 2 formulations seem to suggest that $O$-LAR is more effective than L-SR in the treatment of acromegaly (14-18). N ot enough information is available about the effects of the 2 drugs on glucose control.

\section{MATERIALS AND METHODS \\ Patients}

Ten acromegalic patients ( 6 men and 4 women; age mean $\pm S D$, range: $46 \pm 16,25-68 \mathrm{yr}$; BMI: $29 \pm 5,22.6-40 \mathrm{~kg} / \mathrm{m}^{2}$, body fat percentage [\% BF]: $20 \pm 10,8-36 \%$ ) with active disease were included in the study. Demographic, clinical and hormonal data are reported in Table 1 . At the time of diagnosis, 7 patients had macroadenoma, with extrasellar extension in 4 of them, and 3 
C. Ronchi, P. Epaminonda, V. Cappiello, et al.

(J. Endocrinol. Invest. 25: 502-507, 2002). ${ }^{\circ 2} 2002$, Editrice Kurtis

Table 1 - Clinical and hormonal details of the 10 acromegalic patients before medical treatment.

\begin{tabular}{lccccc}
\hline Patients (sex/age) & $\mathrm{BMI}\left(\mathrm{kg} / \mathrm{m}^{2}\right)$ & Previous treatment & $\mathrm{GH}(\mu \mathrm{g} / \mathrm{l})$ & $\mathrm{IGF}-\mathrm{I}(\mathrm{nmol} / \mathrm{l})$ & Glucose status \\
\hline 1. (M/37) & 26.4 & $\mathrm{~S}$ & 14.0 & 78.6 & Euglycemic \\
2. (M/53) & 22.6 & $\mathrm{~S}, \mathrm{RT}$ & 6.0 & 64.0 & Euglycemic \\
3. $(\mathrm{F} / 48)$ & 34.5 & $\mathrm{~S}, \mathrm{RT}$ & 7.2 & 70.6 & Euglycemic \\
4. $(\mathrm{M} / 40)$ & 29.6 & $\mathrm{~S}, \mathrm{RT}(\gamma-\mathrm{k})$ & 5.2 & 70.2 & Euglycemic \\
5. $(\mathrm{F} / 25)$ & 26.6 & $\mathrm{~S}, \mathrm{RT}(\gamma-\mathrm{k})$ & 16.2 & 83.7 & Euglycemic \\
6. $(\mathrm{M} / 34)$ & $\mathrm{S}$ & $\mathrm{None}$ & 19.1 & 190.0 & Euglycemic \\
7. $(\mathrm{F} / 68)$ & 30.5 & $\mathrm{~S}, \mathrm{RT}$ & 8.3 & 89.2 & Diabetic \\
8. $(\mathrm{M} / 52)$ & 25.5 & $\mathrm{None}$ & 9.4 & 152.5 & Diabetic \\
9. $(\mathrm{M} / 49)$ & 40.0 & $\mathrm{~S}$ & 8.0 & 125.0 & Euglycemic \\
10. $(\mathrm{F} / 50)$ & 29.4 & 22.7 & 6.0 & 67.0 & Euglycemic \\
\hline $\mathrm{RT}$ & $\mathrm{radi}$ & &
\end{tabular}

RT: radiotherapy; S: surgery; $\gamma$-k: gamma-knife; $\mathrm{M}$ : male; F: female.

had microadenoma. Mean duration of acromegaly from time of diagnosis was $76 \pm 48$ months (range 24-180). Eight of 10 patients underwent unsuccessful pituitary surgery followed by pituitary irradiation (conventional external irradiation in 3 and gamma-knife in 2 patients) in 5, from 1 to $5 \mathrm{yr}$ (median: $3 \mathrm{yr}$ ) before the beginning of the study. Before medical therapy, mean GH levels were $10 \pm 5 \mu \mathrm{g} / \mathrm{l}$ (range 5.2-19) and mean IGF-I levels were $99 \pm 43 \mathrm{nmol} / \mathrm{l}$ (range 64-190). O ne patient was also hyperprolactinemic, but did not receive dopaminergic treatment before and during the study. No deficiencies of anteropituitary hormones were found. Two patients had Type 2 diabetes mellitus (DM), according to the American Diabetes Association criteria (19), diagnosed before the start of the study. One patient was treated with oral antidiabetic drugs and the other with both oral antidiabetic drugs and insulin. Eighteen sex- and age-matched healthy subjects, recruited among medical staff (mean BMI $25 \pm 4$ $\mathrm{kg} / \mathrm{m}^{2}$, range 20.1-30), were studied as normal controls for the evaluation of IR. The local Ethical Committee had approved the protocol study, and all the patients gave their informed written consent to participate in this study.

\section{Treatment protocol}

All patients received L-SR for a mean period of $19 \pm 16$ months (range 6-60) as first treatment. The starting schedule of $30 \mathrm{mg}$ im injection every 14 days was adjusted according to GH/IGF-I values, by shortening the interval between drug administration to 10 or 7 days.

After a 3-month washout period, all patients received O-LAR 20 $\mathrm{mg}$ im every 28 days for a mean period of $21 \pm 10$ months (range 6-36). The dose was increased or decreased on the base of the GH/IGF-I levels of each patient.

Fasting serum $\mathrm{GH}$ (mean of at least 5 samples obtained during $2 \mathrm{~h}$ saline infusion) and serum IGF-I levels were evaluated at baseline and at the end of the treatment period with the given somatostatin analogs, on the same day and just before drug injection. Carbohydrate metabolism was checked in all patients by fasting glucose and insulin levels, glycosylated hemoglobin $\left(\mathrm{HbA}_{1 \mathrm{c}}\right)$ levels, IR evaluated by the homeostatic model assessment (HOMA-IR). O nly in non-diabetic patients, serum glucose and insulin response to OGTT were also evaluated. Acromegalyrelated signs and symptoms and side-effects were scored for each patient according to the following scale: 0 : absent; 1 : improved; 2: unchanged; 3 : worsened. Main biochemical and hematological evaluations, like liver and kidney function parameters, blood counts, coagulation factors, and lipid metabolism parameters, were evaluated at baseline and during medical therapy. Ultrasound scans of the gall bladder were also periodically performed.

\section{Methods}

Body composition was evaluated by whole body bioelectrical impedance analysis (BIA), using a portable impedance analyzer (RJ L Systems, U.S.A.). Body fat percentage (\%BF) was calculated using Segal's regression equation (20) and the results compared with those reported by Pichard et al. (21) in healthy subjects matched for age and sex.

Serum $\mathrm{GH}$ levels were measured by an immunofluorimetric assay method supplied by AutoDelfia kit, Wallac OY, Finland. The sensitivity is $0.01 \mu \mathrm{g} / \mathrm{l}$, the intra- and inter-assay coefficients of variation are $2 \%$ and $1.7 \%$ respectively.

Serum IGF-I was measured by a RIA with a commercial kit supplied by Mediagnost, Tübingen, Germany, able to measure total IGF-I by separating IGF-I from IGF binding protein by acidification in IGF-II excess. IGF-II cross-reactivity is less than $0.05 \%$; the intra- and inter-assay coefficient of variation is $3.2 \%$ and $7.4 \%$ respectively. The normal values range from $20-61 \mathrm{nmol} / \mathrm{l}$ at 18 to $6-26 \mathrm{nmol} / \mathrm{l}$ at $70 \mathrm{yr}$.

Fasting glucose was measured with standard methods (enzymatic method, intra- and inter-assay coefficients of variation are $2.1 \%$ and $2.6 \%$, respectively). Insulin concentrations were assessed by an immunoenzymetric one-step assay (Medgenics Diagnostics, Belgium); its sensitivity is $0.15 \mu \mathrm{U} / \mathrm{ml}$; intra- and inter-assay coefficients of variation are $4 \%$ and $7.5 \%$, respectively.

Insulin resistance was determined using the homeostate model assessment (HOMA) method (22). This is a computer-solved model used to predict the degree of IR starting from fasting plasma insulin ( $\mathrm{Fl})$ and glucose concentrations (FG): IR=FI ( $\mathrm{mU} / \mathrm{l})$ x FG (mmol/l)/ 22.5. Comparison of a patient's fasting values with the model predictions allows a quantitative assessment of insulin resistance to the hyperglycemia. As recently reported, it closely mirrors the euglycemic and hyperglycemic clamp technique in the assessment of IR and strongly predicts the development of Type 2 DM (23-25). 


\section{Statistical analyses}

All results are expressed as mean \pm SD. As data are not normally distributed, a non-parametric test (Wilcoxon's rank sum test) was used for multiple comparisons among group means. The AUCs of glucose and insulin during OGTT were estimated according to the trapezoidal method. Chi square test was used to compare clinical modifications and percentage of patients that normalize GH and IGF-I levels.

Values of $p<0.05$ were considered statistically significant.

\section{RESULTS}

\section{Effects on glucose metabolism}

Glucose and insulin levels evaluated in all patients in fasting conditions and after OGTT were similar before the beginning of both treatments.

In the 8 euglycemic patients, baseline mean fasting glucose levels were $83 \pm 9.5 \mathrm{mg} / \mathrm{dl}$ and did not change significantly during L-SR treatment, while during $0-L A R$ treatment increased to $104 \pm 27$ $\mathrm{mg} / \mathrm{dl}$ ( $p<0.05$ vs baseline, L-SR and healthy subjects). Mean serum $\mathrm{HbA}_{1 \mathrm{c}}$ levels increased, but remained in the normal range, only during $0-L A R$ therapy, from $5 \pm 0.4 \%$ to $5.5 \pm 0.5 \%$ ( $p<0.05$ vs baseline, L-SR and control subjects). Furthermore, the glucose response to the OGTT, measured as AUC value, did not change after L-SR (from $18 \pm 3$ to $19.7 \pm 2 \mathrm{~g} / \mathrm{dl} / 120 \mathrm{~min})$, but increased after 0 LAR (to $22.4 \pm 4 \mathrm{~g} / \mathrm{dl} / 120 \mathrm{~min} ; \mathrm{p}=0.05$ vs baseline and $p<0.05$ vs L-SR) (Fig. 1). Mean blood glucose peak levels at $2 \mathrm{~h}$ increased only during 0 -LAR from $107 \pm 34$ to $121 \pm 34 \mathrm{mg} / \mathrm{dl}(p=N S)$. Two of the 8 non-diabetic patients developed an impaired glucose tolerance after 6 months of 0 -LAR treatment.

Basal fasting insulin levels were higher than in healthy controls $(16 \pm 13$ vs $7.4 \pm 5.5 \mu \mathrm{U} / \mathrm{m}, \mathrm{p}<0.05)$ and decreased to $8 \pm 4.4 \mu \mathrm{U} / \mathrm{ml}(\mathrm{p}<0.05$ vs baseline, NS vs healthy subjects) during L-SR and to $6.8 \pm 2$ $\mu \mathrm{U} / \mathrm{ml}(\mathrm{p}<0.05$ vs baseline, NS vs L-SR and control

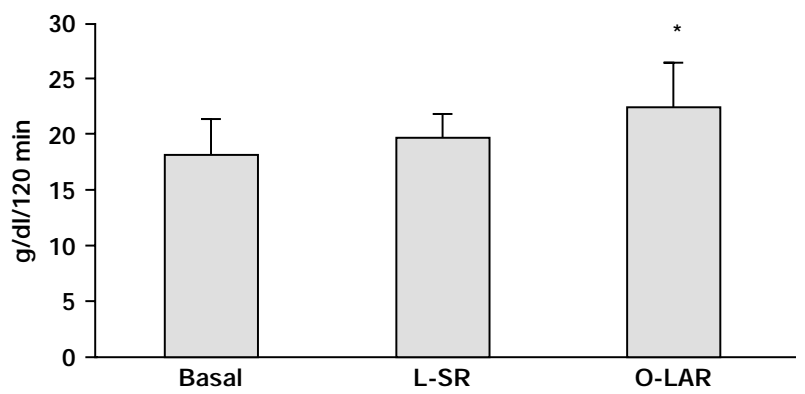

Fig. 1 - Glucose AUC levels in 8 acromegalic patients before and after chronic medical treatment with lanreotide slow release (L-SR) and octreotide-long acting release (O-LAR). ${ }^{*} p<0.05$ vs L-SR.

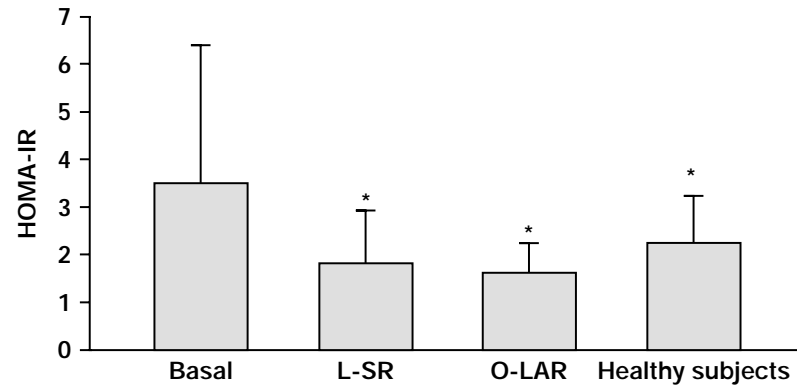

Fig. 2 - Homeostatic model assessment of insulin resistance (HOMA-IR) levels in healthy subjects and in acromegalic patients before and after medical treatment. L-SR: lanreotide slow release; 0 -LAR: octreotide-long acting release. ${ }^{*} p<0.05$ vs acromegalic patients at baseline.

subjects), during $\mathrm{O}$-LAR. Expressed as AUC during OGTT, serum insulin levels decreased from $10 \pm 7$ to $6 \pm 2.2 \mathrm{mU} / \mathrm{l} / 120 \mathrm{~min}$ during L-SR therapy and to $3.8 \pm 1.8 \mathrm{mU} / / / 120 \mathrm{~min}(p<0.05$ vs baseline and $p$ NS vs L-SR) during 0 -LAR therapy.

Mean HOMA-IR, which is an IR index, was at baseline 3.5 \pm 2.9 (range 0.5-10), significantly higher than in healthy control subjects (2.2 \pm 1 , range 0.4-3.1). It decreased during both L-SR and O-LAR therapy to $1.8 \pm 1.1$ (range $0.2-2.6)(p<0.05$ vs baseline) and to $1.6 \pm 0.6$ (range $1.1-1.8$ ) respectively ( $p<0.05$ vs baseline) (Fig. 2). No correlation between HOMA-IR and BMI ( $29 \pm 5.5$ to $27.9 \pm 6 \mathrm{~kg} / \mathrm{m}^{2}$ in L-SR to $29.2 \pm 8$ $\mathrm{kg} / \mathrm{m}^{2}$ in O-LAR or \%BF) before and after both kind of treatments was found, demonstrating that the decrease of IR is not associated to a decrease of fat mass.

All data about carbohydrate metabolism in euglycemic patients are reported in Table 2 .

In 1 of the 2 diabetic patients no variation in fasting glucose and $\mathrm{HbA}_{1 \mathrm{c}}$ levels was observed during $\mathrm{L}$ SR treatment, while in the other fasting glucose lev-

Table 2 - Effects of the medical therapy on glucose metabolism in 8 euglycemic acromegalic patients: percentage of variation.

\begin{tabular}{lcc}
\hline & L-SR & O-LAR \\
\hline Fasting glucose & $-2 \pm 13 \%$ & $20 \pm 12 \% *, * *$ \\
Fasting insulin & $-42 \pm 18 \% *$ & $-46 \pm 31 \% *$ \\
Glucose AUC & $12 \pm 20 \%$ & $28 \pm 32 \% *, * *$ \\
Insulin AUC & $-39 \pm 32 \% *$ & $-56 \pm 14 \% *$ \\
HbA $_{1 \mathrm{C}}$ & $0 \%$ & $10 \pm 6 \% *, * *$ \\
HOMA-IR & $-42 \pm 23 \% *$ & $-34 \pm 37 \% *$
\end{tabular}

*p $<0.05$ vs basal; $* * p<0.05$ vs L-SR. HOMA-IR: homeostatic model assessment of insulin resistance; L-SR: lanreotide slow release; O-LAR: OCtreotide long acting release. 
els decreased from 234 to $150 \mathrm{mg} / \mathrm{dl}$ with a reduction in $\mathrm{HbA}_{1 c}$ serum levels from 10 to $7.3 \%$, without any change in weight, lifestyle, oral antidiabetic and insulin therapy respectively. During 0 -LAR treatment, both patients had a worsening in fasting glucose (to 165 and $250 \mathrm{mg} / \mathrm{dl}$ ) and $\mathrm{HbA}_{1 \mathrm{c}}$ levels (to 7.2 and $7.8 \%$ ) and requested an increase in oral antidiabetic and/or in insulin doses. No correlation between the effects on glucose tolerance and the different dose of L-SR or O-LAR was found.

Effects on GH and IGF-I levels

During L-SR therapy, mean serum GH concentrations decreased from $10 \pm 5$ to $4.2 \pm 2.7 \mu \mathrm{g} / \mathrm{l}(\mathrm{p}<0.05)$ and 4 of 10 patients reached $\mathrm{GH}$ values below 2.5 $\mu \mathrm{g} / \mathrm{l}$.

During O-LAR therapy, mean $\mathrm{GH}$ levels decreased from $9.8 \pm 5$ to $3.1 \pm 2.4 \mu \mathrm{g} / \mathrm{l}(\mathrm{p}<0.05)$ and were suppressed below $2.5 \mu \mathrm{g} / \mathrm{l}$ in 5 out of 10 patients.

Mean GH levels were significantly lower with 0 -LAR treatment than with L-SR $(p<0.05)$.

Serum IGF-I levels fell from $99 \pm 43$ to $66 \pm 27 \mathrm{nmol} / \mathrm{l}$ $(p<0.05)$ during L-SR therapy and from $98.5 \pm 41$ to $57 \pm 25 \mathrm{nmol} / \mathrm{l}(p<0.05)$ during 0 -LAR therapy. Differences between IGF-I levels when receiving L-SR and 0 -LAR did not reach statistical significance. Normal age-adjusted IG F-I concentrations were reached in 1 and in 2 patients with L-SR and 0-LAR respectively.

\section{Clinical response and side-effects}

The number of patients that reported acromegalic-related symptoms decreased during medical therapy respect to baseline ( $p=N S$ L-SR vs baseline, $p<0.050$-LAR vs baseline for headache, paraesthesia and sweating, $p=N S$ L-SR vs $0-L A R)$. Furthermore, the administration of 0 -LAR resulted in an improvement of the symptom scores in headache and arthralgia, while the other symptoms completely disap peared. Both drugs were well tolerated. Side-effects, like abdominal pain (2 patients), diarrhea (4 patients), epigastralgia (1 patient) and emesis (1 patient), present during L-SR treatment were mild and transient and further decreased during 0 -LAR treatment. At ultrasound examination, no gallstone was observed either before or during medical therapy. No changes in routine hematological and biochemical tests were found.

\section{DISCUSSION}

Somatostatin analogs, L-SR and O-LAR, represent the medical therapy of choice in acromegaly. They demonstrated to be effective in normalizing $\mathrm{GH}$ and IGF-I levels and are generally well tolerated (813). In this study, during O-LAR treatment a more important improvement in acromegaly related signs and symptoms and lower means serum GH and IGF-I concentrations with respect to L-SR were observed. Furthermore, a greater proportion of patients reached $\mathrm{GH}$ levels less than $2.5 \mu \mathrm{g} / \mathrm{l}$ and/or normal IGF-I levels in agreement with previous published data (14-18). 0-LAR was also better tolerated by the patients because of less abdo minal sideeffects and the reduction in the frequency of injections.

The non-diabetic acromegalic patients had basal fasting insulin levels and basal HOMA-IR, an IR index, significantly higher than sex- and age-matched normal subjects. Both of these parameters significantly decreased during medical therapy and became similar to normal subjects values. Comparable conclusions were drawn by Kasayama et al. (26). In fact, they reported that 11 acromegalic patients had increased IR, evaluated by HOMA, before surgery that normalized after successfully surgical treatment. Previous studies about octreotide treatment reported an improvement in whole body insulin sensitivity with variable effects on carbohydrate metabolism. An increase in fasting glucose levels was more frequently found in previously euglycemic patients, while glucose response in diabetic patients was totally unpredictable (4-7).

The comparison between the effects of the 2 new long-acting somatostatin analogs revealed higher glucose and lower insulin levels during the treatment with O-LAR with respect to L-SR. Other Authors did not report any difference in the effects of the 2 formulations on fasting glucose and $\mathrm{HbA}_{1 \mathrm{c}}$ levels, but none of them measured glucose and insulin response to OGTT $(14,15)$. In diabetic patients we noted impairment in fasting glucose and $\mathrm{HbA}_{1 \mathrm{c}}$ levels and an increase in oral antidiabetic and/or insulin regimen only during 0 -LAR treatment. The interpretation of these differences between the 2 drugs is not simple. It is known that L-SR and OLAR act by coupling to the same pituitary somatostatin receptors of subtype 2 and 5 , showing similar but not identical affinity, especially for the last one (27). Furthermore, receptor subtype 5 strongly co-localizes with insulin in the pancreatic $\beta$-cell (28). So, the chance of a more selective action of 0 -LAR on subtype 5 can be one of the possible explications for the effect of $O$-LAR treatment on insulin and glucose levels. Another possibility is that the greater power of 0 -LAR formulation in decreasing $\mathrm{GH}$ secretion applies to insulin too. This may result in lower insulin levels and in a deterio- 
ration of glucose control in insulin-resistant patients that are able to maintain normal-glycemia only with high insulin levels.

As far as HOMA-IR is concerned, some considerations have to be done. In fact, in acromegaly the basal increase of HOMA-IR is not related to the elevated BMI. In this disease, IR status is prevalently due to hepatic and peripheral direct metabolic effects of the high $\mathrm{GH}$ levels, rather than to obesity $(29,30)$. Furthermore, acromegalic patients have altered body composition characterized by increased lean body mass and reduced fat mass compared to control subjects with the same BMI (31) whereas our patients had normal \% BF. HOMA-IR significantly decreased after medical therapy, paralleling the decrease of $\mathrm{GH}$, while $\mathrm{BMI}$ remained stable. The HOMA-IR decrease was similar with the 2 types of treatments, even if 0 -LAR seems to induce a larger suppression of basal $\mathrm{Fl}$ levels which may be responsible for the associated worsening of FG levels. Somatostatin analogs have complex effects on glucose control, as they inhibit contemporary $\mathrm{GH}$, insulin and glucagon secretion. These effects are only partially valuable with HOMA method, which is just based on 2 variables as fasting glycemia and insulinemia.

In summary, the choice of the right somatostatin analog has to be done carefully owing to the lack of considerable endocrine selectivity. O-LAR seems to be more effective in medical therapy of acromegaly and better tolerated by patients, but it seems also to affect more glucose metabolism, both in previously euglycemic and in diabetic patients. Therefore, in patients with impaired glucose tolerance or DM treatment with L-SR is the first choice, while in non-diabetic patients treatment with 0 -LAR is preferable.

\section{ACKNOWLEDGEMENTS}

The Authors wish to thank M rs. Rita Deriu and Dr. Giuseppe Ivan Vaghi for their kind contributions and technical assistance. The present study was partially supported by research grants 9906153187-00 from MURST, Rome and from Ospedale Maggiore IRCCS (Milan).

\section{REFERENCES}

1. Nabarro J.D.N. Acromegaly. Clin. Endocrinol. 1987, 26: 481-512.

2. Melmed S. Acromegaly. N. Engl. J. Med. 1990, 322: 966977.

3. Holdaway I.M., Rajasoorya C. Epidemiology of acromegaly. Pituitary 1999, 2: 29-41.
4. Ho K., Weissberger A., Marbach P., Lazarus L. Therapeutic efficacy of somatostatin analog SM S 201-995 (octreotide) in acromegaly. Ann Intern. Med. 1990, 112: 173-181.

5. Koop B.L., Harris A.G., Ezzat S. Effect of octreotide on glucose tolerance in acromegaly. Eur. J. Endocrinol. 1994, 130: 581-586.

6. Arosio M., Macchelli S., Rossi C.M., Casati G., Biella O., Faglia G. Effects of treatment with octreotide in acromegalic patients- a multicenter Italian study. Eur. J. Endocrinol. 1995, 133: 922-928.

7. Arosio M., Sartore G., Rossi C.M., Casati G., Faglia G., Manzato E. Italian Multicenter O ctreotide Study Group. LDL physical properties, lipoprotein and Lp(a) levels in acromegalic patients. Effects of octreotide therapy. Atherosclerosis 2000, 151: 551-557.

8. Stewart P.M., Kane K.F., Stewart S.E., Lancranjan I., Sheppard M.C. Depot long-acting somatostatin analog is an effective treatment for acromegaly. J . Clin. Endocrinol. Metab. 1995, 80: 3267-3272.

9. Davies P.H., Stewart P.E., Lancranjan I., Sheppard M.C., Stewart P.M. Long term therapy with long-acting octreotide (Sandostatin LAR) for the management of acromegaly. Clin. Endocrinol. 1998, 48: 311-316.

10. Flogstad A.K., Halse J ., Bakke S., et al. Sand ostatin LAR in acromegalic patients: long term treatment. J. Clin. Endocrinol. Metab. 1997, 82: 23-28.

11. Giusti M., Gussoni G., Cuttica C., Giordano G. Effectiveness and tolerability of slow release lanreotide treatment in active acromegaly: six months report on an Italian multicenter study. J. Clin. Endocrinol. M etab. 1996, 81: 20892097.

12. Caron P., Morange I., Cogne M., J aquet P. Three year follow-up of acromegalic patients treated with intramuscolar slow-release Lanreotide. J. Clin. Endocrinol. Metab. 1997, 82: 18-22.

13. Verhelst J.A., Pedroncelli A.M., Abs R., et al. Slow-release lanreotide in the treatment of acromegaly: a study in 66 patients. Eur. J. Endocrinol. 2000, 143: 577-584.

14. Turner H.E., Vadivale A., Keenan J., Wass J .A.D. A comparison of lanreotide and octreotide LAR for treatment of acromegaly. Clin Endocrinol. 1999, 51: 275-280.

15. Cozzi R., Dallabonzana D., Attanasio R., Barausse M., O ppizzi G. A comparison between octreotide-LAR and lanreotide-SR in the chronic treatment of acromegaly. Eur. J . Endocrinol. 1999, 141: 267-271.

16. Chanson P., Boerlin V., Ajzenberg C., et al. Comparison of octreotide acetate LAR and lanreotide SR in patients with acromegaly. Clin. Endocrinol. 2000, 53: 577-586.

17. J enkins P.J ., Akker S., Chew S.L., Besser G.M., Monson J .P., Grossman A.B. O ptimal dosage interval for depot somatostatin analog therapy in acromegaly requires individual titration. Clin. Endocrinol. 2000, 53: 719-724.

18. Kendall-Taylor P., Miller M., Gebbie J ., Turner S., AlMaskari M. Long acting octreotide LAR compared with lanreotide SR in the treatment of acromegaly. Pituitary 2000, 3: 61-65. 
C. Ronchi, P. Epaminonda, V. Cappiello, et al.

(J. Endocrinol. Invest. 25: 502-507, 2002). ${ }^{\circ 2002}$, Editrice Kurtis

19. The expert committee on the diagnosis and classification of the diabetes mellitus. Committee report. Diabetes Care 1997, 20: 1183-1197.

20. Segal K.R., Van Loan M., Fitzgerald P.I., Hodgdon J.A., Van Itallie T.B. Lean body mass estimation by bioelectrical impedance analysis: a four-site cross validation study. Am. J. Clin. Nutr. 1988, 47: 7-14.

21. Pichard C., Kyle U.G., Bracco D., Slosman D.O., Morabia A., Schutz Y. Reference values of fat-free and fat masses by bioelectrical impedance analysis in 3393 healthy subjects. Nutrition 2000; 16: 245-254.

22. Matthews D.R., Hosker J .P., Rudenski A.S., Naylor B.A., Treacher D.F., Turner R.C. Homeostasis model assessment: insulin resistance and $\beta$-cell function from fasting plasma glucose and insulin concentration in man. Diabetologia 1985, 28: 412-419.

23. Haffner S.M., Gonzales C., M iettinen H., Kennedy E., Stern M.P. A prospective analysis of the HOMA model. Diabetes Care 1996, 10: 1138-1143.

24. The Mexico City Diabetes Study. A prospective analysis of the HOMA model. Diabetes Care 1996, 19: 1138-1143.

25. Bonora E., Targher G., Alberiche M., et al. Homeostasis model assessment closely mirrors the glucose clamp technique in the assessment of insulin sensitivity: studies in subjects with various degrees of glucose tolerance and insulin sensitivity. Diabetes Care 2000, 23: 57-63.

26. Kasayama S., O tsuki M., Takagi M., et al. Impaired $\beta$-cell function in the presence of reduced insulin sensitivity determines glucose tolerance status in acromegalic patients. Clin. Endocrinol. 2000, 52: 549-555.

27. Reisine T., Bell G.I. Molecular biology of somatostatin receptors. Endocr. Rev. 1995, 16: 427-444.

28. Mitra S., Mezey E., Hunyady B., et al. Colocalization of somatostatin receptor sst5 and insulin in rat pancreatic $\beta$ cells. Endocrinology 1999, 140: 3790-3796.

29. Scelles V., Raccah D., Alessi M.C., Vialle J.M., J uhan-Vague I., Vague P. Plasminogen activator inhibitor 1 and insulin levels in various insulin resistance status. Diabetes Metab. 1992, 18: 38-42.

30. Moller N., Schmitz O., J oorgensen J.O., et al. Basal and insulin-stimulated substrate metabolism in patients with active acromegaly before and after adenomectomy. J . Clin. Endocrinol. Metab. 1992, 74: 1012-1019.

31. Brummer R.J .M., Lonn L., Kvist H., Grangard U., Bengtsson B.A., Sjöström L. Adipose tissue and muscle volume determination by computed tomography in acromegaly, before and 1 year after adenomectomy. Eur. J. Clin. Invest. 1993, 23: 199-205. 